\title{
Canonical matrices with entries integers modulo $p$
}

\author{
Krasimir Yordzhev \\ Faculty of Mathematics and Natural Sciences \\ South-West University "Neofit Rilski" \\ Blagoevgrad, Bulgaria \\ e-mail: yordzheveswu.bg
}

Received: 27 June 2018

Accepted: 27 October 2018

\begin{abstract}
The work considers an equivalence relation in the set of all $n \times m$ matrices with entries in the set $[p]=\{0,1, \ldots, p-1\}$. In each element of the factor-set generated by this relation, we define the concept of canonical matrix, namely the minimal element with respect to the lexicographic order. We have found a necessary and sufficient condition for an arbitrary matrix with entries in the set $[p]$ to be canonical. For this purpose, the matrices are uniquely represented by ordered $n$-tuples of integers.
\end{abstract}

Keywords: Permutation matrix, Weighing matrix, Hadamard matrix, Semi-canonical matrix, Canonical matrix, Ordered $n$-tuples of integers.

2010 Mathematics Subject Classification: 05B20, 15B36.

\section{Introduction and notation}

This paper presents a generalization and an improvement of the results obtained in [7].

Let $k$ and $p$ be integers, $k \leq p$. By $[k, p]$ we denote the set

$$
[k, p]=\{k, k+1, \ldots, p\}
$$

and by $[p]$ the set

$$
[p]=[0, p-1]=\{0,1,2, \ldots, p-1\} .
$$

With $\mathcal{M}_{n \times m}^{p}$ we will denote the set of all $n \times m$ matrices with entries in the set $[p]$.

When $p=2$, a matrix whose entries belong to the set $[2]=\{0,1\}$ is called binary (or boolean, or $(0,1)$-matrix). 
When $p=3$, a $n \times n$ matrix $H$ whose entries belong to the set $\{1,-1\} \equiv\{1,2\}(\bmod 3)$ is Hadamard if $H H^{T}=n I_{n}$, where $H^{T}$ is the transposed matrix of $H$ and $I_{n}$ is the $n \times n$ identity matrix. It is well known that $n$ is necessarily 1,2 , or a multiple of four $[2,3]$.

When $p=3$, a $n \times n$ matrix $W$ whose entries belong to the set $\{0,1,-1\} \equiv\{0,1,2\}(\bmod 3)$ is weighing matrix of order $n$ with weight $k$, if $W W^{T}=k I_{n}$. For more information on applications of weighing matrices, we refer the reader to [4]. A $n \times n$ weighing matrix $W$ with weight $k$ is Hadamard if $k=n$ (see [1]).

A square binary matrix is called a permutation matrix, if there is exactly one 1 in every row and every column. Let us denote the group of all $n \times n$ permutation matrices by $\mathcal{P}_{n}$. It is well known (see $[5,6])$ that the multiplication of an arbitrary real or complex matrix $A$ from the left with a permutation matrix (if the multiplication is possible) leads to permutation of the rows of the matrix $A$, while the multiplication of $A$ from the right with a permutation matrix leads to permutation of the columns of $A$.

A transposition is a matrix obtained from the $n \times n$ identity matrix $I_{n}$ by interchanging two rows or two columns. With $\mathcal{T}_{n} \subset \mathcal{P}_{n}$ we denote the set of all transpositions in $\mathcal{P}_{n}$, i.e., the set of all $n \times n$ permutation matrices, which multiplying from the left an arbitrary $n \times m$ matrix swaps the places of exactly two rows, while multiplying from the right an arbitrary $k \times n$ matrix swaps the places of exactly two columns.

Definition 1.1. Let $A, B \in \mathcal{M}_{n \times m}^{p}$. We will say that the matrices $A$ and $B$ are equivalent and we will write

$$
A \sim B,
$$

if there exist permutation matrices $X \in \mathcal{P}_{n}$ and $Y \in \mathcal{P}_{m}$, such that

$$
A=X B Y \text {. }
$$

In other words, $A \sim B$, if $A$ is received from $B$ after a permutation of some of the rows and some of the columns of $B$. Obviously, the introduced relation is an equivalence relation.

In each element of the factor-set generated by the relation " " described in Definition 1.1, we define the concept of canonical matrix, namely the minimal element with respect to the lexicographic order. For this purpose, the matrices are uniquely represented by ordered $n$-tuples of integers. The purpose of this work is to get a necessary and sufficient condition for an arbitrary matrix with entries in the set $[p]$ to be canonical. This task is solved in the particular case where $p=2$ in [7]. The case where $p=3$ will be useful in classification of Hadamard matrices and weighing matrices.

\section{Representation of matrices from $\mathcal{M}_{n \times m}^{p}$ via ordered $n$-tuples of integers}

Let $A=\left(a_{i j}\right)_{n \times m} \in \mathcal{M}_{n \times m}^{p}, 1 \leq i \leq n, 1 \leq j \leq m$ and let

$$
x_{i}=\sum_{j=1}^{m} a_{i j} p^{m-j}, i=1,2, \ldots n .
$$


Obviously

$$
0 \leq x_{i} \leq p^{m}-1 \quad \text { for every } i=1,2, \ldots n
$$

and $x_{i}$ is a natural number written in notation in the number system with the base $p$ whose digits are consistently the entries of the $i$-th row of $A$.

With $r(A)$ we will denote the ordered $n$-tuple

$$
r(A)=\left\langle x_{1}, x_{2}, \ldots, x_{n}\right\rangle .
$$

Similarly, with $c(A)$ we will denote the ordered $m$-tuple

$$
c(A)=\left\langle y_{1}, y_{2}, \ldots, y_{m}\right\rangle,
$$

where

$$
y_{j}=\sum_{i=1}^{n} a_{i j} p^{n-i}, \quad 0 \leq y_{j} \leq p^{n}-1, \quad j=1,2, \ldots m
$$

and $y_{j}$ is a natural number written in notation in the number system with the base $p$ whose digits are consistently the entries of the $i$-th column of $A$.

It is easy to see that for every $A \in \mathcal{M}_{n \times m}^{p}, c(A)=r\left(A^{T}\right)$ and $r(A)=c\left(A^{T}\right)$, where $A^{T}$ is the transposed matrix of $A$.

We consider the sets:

$$
\begin{aligned}
\mathcal{R}_{n \times m}^{p} & =\left[0, p^{m}-1\right]^{n} \\
& =\left\{\left\langle x_{1}, x_{2}, \ldots, x_{n}\right\rangle \mid 0 \leq x_{i} \leq p^{m}-1, i=1,2, \ldots n\right\} \\
& =\left\{r(A) \mid A \in \mathcal{M}_{n \times m}^{p}\right\}
\end{aligned}
$$

and

$$
\begin{aligned}
\mathcal{C}_{n \times m}^{p} & =\left[0, p^{n}-1\right]^{m} \\
& =\left\{\left\langle y_{1}, y_{2}, \ldots, y_{m}\right\rangle \mid 0 \leq y_{j} \leq p^{n}-1, j=1,2, \ldots m\right\} \\
& =\left\{c(A) \mid A \in \mathcal{M}_{n \times m}^{p}\right\}
\end{aligned}
$$

Thus, we define the following two mappings:

$$
r: \mathcal{M}_{n \times m}^{p} \rightarrow \mathcal{R}_{n \times m}^{p}
$$

and

$$
c: \mathcal{M}_{n \times m}^{p} \rightarrow \mathcal{C}_{n \times m}^{p}
$$

which are bijective and therefore

$$
\mathcal{R}_{n \times m}^{p} \cong \mathcal{M}_{n \times m}^{p} \cong \mathcal{C}_{n \times m}^{p}
$$

We will denote the lexicographic orders in $\mathcal{R}_{n \times m}^{p}$ and in $\mathcal{C}_{n \times m}^{p}$ with " $<$ ".

Example 2.1. Let

$$
A=\left(\begin{array}{llll}
1 & 0 & 3 & 2 \\
0 & 2 & 1 & 0 \\
0 & 1 & 1 & 3
\end{array}\right) \in \mathcal{M}_{3 \times 4}^{4}
$$


Then

$$
\begin{gathered}
x_{1}=1 \cdot 4^{3}+0 \cdot 4^{2}+3 \cdot 4^{1}+2 \cdot 4^{0}=1 \cdot 64+0 \cdot 16+3 \cdot 4+2 \cdot 1=78, \\
x_{2}=0 \cdot 4^{3}+2 \cdot 4^{2}+1 \cdot 4^{1}+0 \cdot 4^{0}=0 \cdot 64+2 \cdot 16+1 \cdot 4+0 \cdot 1=36, \\
x_{3}=0 \cdot 4^{3}+1 \cdot 4^{2}+1 \cdot 4^{1}+3 \cdot 4^{0}=0 \cdot 64+1 \cdot 16+1 \cdot 4+3 \cdot 1=23, \\
y_{1}=1 \cdot 4^{2}+0 \cdot 4^{1}+0 \cdot 4^{0}=1 \cdot 16+0 \cdot 4+0 \cdot 1=16, \\
y_{2}=0 \cdot 4^{2}+2 \cdot 4^{1}+1 \cdot 4^{0}=0 \cdot 16+2 \cdot 4+1 \cdot 1=9, \\
y_{3}=3 \cdot 4^{2}+1 \cdot 4^{1}+1 \cdot 4^{0}=3 \cdot 16+1 \cdot 4+1 \cdot 1=53, \\
y_{4}=2 \cdot 4^{2}+0 \cdot 4^{1}+3 \cdot 4^{0}=2 \cdot 16+0 \cdot 4+3 \cdot 1=35, \\
r(A)=\langle 78,36,23\rangle, \\
c(A)=\langle 16,9,53,35\rangle .
\end{gathered}
$$

Theorem 2.1. Let $A$ be an arbitrary matrix from $\mathcal{M}_{n \times m}^{p}$. Then:

a) If $X_{1}, X_{2}, \cdots, X_{s} \in \mathcal{T}_{n}$ are such that

$$
r\left(X_{1} X_{2} \ldots X_{s} A\right)<r\left(X_{2} X_{3} \ldots X_{s} A\right)<\cdots<r\left(X_{s-1} X_{s} A\right)<r\left(X_{s} A\right)<r(A)
$$

then

$$
c\left(X_{1} X_{2} \ldots X_{s} A\right)<c(A)
$$

b) If $Y_{1}, Y_{2}, \cdots, Y_{t} \in \mathcal{T}_{m}$ are such that

$$
c\left(A Y_{1} Y_{2} \ldots Y_{t}\right)<c\left(A Y_{1} Y_{2} \ldots Y_{t-1}\right)<\cdots<c\left(A Y_{1} Y_{2}\right)<c\left(A Y_{1}\right)<c(A),
$$

then

$$
r\left(A Y_{1} Y_{2} \ldots Y_{t}\right)<r(A)
$$

Proof. a) Induction by $s$.

Let $s=1$ and let $X \in \mathcal{T}_{n}$ be a transposition which multiplying an arbitrary matrix $A=\left(a_{i j}\right) \in \mathcal{M}_{n \times m}^{p}$ from the left swaps the places of the rows of $A$ with numbers $u$ and $v$ $(1 \leq u<v \leq n$ ), while the remaining rows stay in their places. In other words, if

$$
A=\left(\begin{array}{cccccc}
a_{11} & a_{12} & \cdots & a_{1 r} & \cdots & a_{1 m} \\
a_{21} & a_{22} & \cdots & a_{2 r} & \cdots & a_{2 m} \\
\vdots & \vdots & & \vdots & & \vdots \\
a_{u 1} & a_{u 2} & \cdots & a_{u r} & \cdots & a_{u m} \\
\vdots & \vdots & & \vdots & & \vdots \\
a_{v 1} & a_{v 2} & \cdots & a_{v r} & \cdots & a_{v m} \\
\vdots & \vdots & & \vdots & & \vdots \\
a_{n 1} & a_{n 2} & \cdots & a_{n r} & \cdots & a_{n m}
\end{array}\right)
$$


then

$$
X A=\left(\begin{array}{cccccc}
a_{11} & a_{12} & \cdots & a_{1 r} & \cdots & a_{1 m} \\
a_{21} & a_{22} & \cdots & a_{2 r} & \cdots & a_{2 m} \\
\vdots & \vdots & & \vdots & & \vdots \\
a_{v 1} & a_{v 2} & \cdots & a_{v r} & \cdots & a_{v m} \\
\vdots & \vdots & & \vdots & & \vdots \\
a_{u 1} & a_{u 2} & \cdots & a_{u r} & \cdots & a_{u m} \\
\vdots & \vdots & & \vdots & & \vdots \\
a_{n 1} & a_{n 2} & \cdots & a_{n r} & \cdots & a_{n m}
\end{array}\right),
$$

where $a_{i j} \in[p]=\{0,1, \ldots, p-1\}, 1 \leq i \leq n, 1 \leq j \leq m$.

Let

$$
r(A)=\left\langle x_{1}, x_{2}, \ldots x_{u-1}, x_{u}, \ldots x_{v-1}, x_{v}, \ldots, x_{n}\right\rangle .
$$

Then,

$$
r(X A)=\left\langle x_{1}, x_{2}, \ldots x_{u-1}, x_{v}, \ldots x_{v-1}, x_{u}, \ldots, x_{n}\right\rangle .
$$

Since $r(X A)<r(A)$, then according to the properties of the lexicographic order $x_{v}<x_{u}$. Let the representation of $x_{u}$ and $x_{v}$ in notation in the number system with the base $p$ (with an eventual addition of unessential zeros in the beginning if necessary) be respectively as follows:

$$
\begin{aligned}
& x_{u}=a_{u 1} a_{u 2} \cdots a_{u r} \cdots a_{u m}, \\
& x_{v}=a_{v 1} a_{v 2} \cdots a_{v r} \cdots a_{v m} .
\end{aligned}
$$

Since $x_{v}<x_{u}$, then there exists an integer $r \in\{1,2, \ldots, m\}$, such that $a_{u j}=a_{v j}$ when $j<r$, and $a_{v r}<a_{u r}$. Hence, if $c(A)=\left\langle y_{1}, y_{2}, \ldots, y_{m}\right\rangle, c(X A)=\left\langle z_{1}, z_{2}, \ldots, z_{m}\right\rangle$, then $y_{j}=z_{j}$ when $j<r$, while the representation of $y_{r}$ and $z_{r}$ in notation in the number system with the base $p$ (with an eventual addition of unessential zeros in the beginning if necessary) is respectively as follows:

$$
\begin{aligned}
& y_{r}=a_{1 r} a_{2 r} \cdots a_{u-1 r} a_{u r} \cdots a_{v r} \cdots a_{n r}, \\
& z_{r}=a_{1 r} a_{2 r} \cdots a_{u-1 r} a_{v r} \cdots a_{u r} \cdots a_{n r} .
\end{aligned}
$$

Since $a_{v r}<a_{u r}$, then $z_{r}<y_{r}$, whence it follows that $c(X A)<c(A)$.

We assume that for every $s$-tuple of transpositions $X_{1}, X_{2}, \ldots, X_{s} \in \mathcal{T}_{n}$ and for every matrix $A \in \mathcal{M}_{n \times m}^{p}$ from

$$
r\left(X_{1} X_{2} \ldots X_{s} A\right)<r\left(X_{2} \cdots X_{s} A\right)<\cdots<r\left(X_{s} A\right)<r(A)
$$

it follows that

$$
c\left(X_{1} X_{2} \ldots X_{s} A\right)<c(A)
$$

and let $X_{s+1} \in \mathcal{T}_{n}$ be such that

$$
r\left(X_{1} X_{2} \ldots X_{s} X_{s+1} A\right)<r\left(X_{2} \cdots X_{s+1} A\right)<\cdots<r\left(X_{s+1} A\right)<r(A) .
$$

According to the above proved $c\left(X_{s+1} A\right)<c(A)$. 
We put

$$
A_{1}=X_{s+1} A \text {. }
$$

According to the induction assumption from

$$
r\left(X_{1} X_{2} \ldots X_{s} A_{1}\right)<r\left(X_{2} \cdots X_{s} A_{1}\right)<\cdots<r\left(X_{s} A_{1}\right)<r\left(A_{1}\right),
$$

it follows that

$$
c\left(X_{1} X_{2} \cdots X_{s} X_{s+1} A\right)=c\left(X_{1} X_{2} \cdots X_{s} A_{1}\right)<c\left(A_{1}\right)=c\left(X_{s+1} A\right)<c(A),
$$

with which we have proven a).

b) is proven similarly to a).

In effect is also the dual to Theorem 2.1 statement, in which instead of the sign " $<$ " everywhere we put the sign " $>$ ".

Theorem 2.2. (Dual theorem) Let $A$ be an arbitrary matrix from $\mathcal{M}_{n \times m}^{p}$. Then:

a) If $X_{1}, X_{2}, \ldots, X_{s} \in \mathcal{T}_{n}$ are such that

$$
r\left(X_{1} X_{2} \ldots X_{s} A\right)>r\left(X_{2} X_{3} \ldots X_{s} A\right)>\ldots>r\left(X_{s-1} X_{s} A\right)>r\left(X_{s} A\right)>r(A)
$$

then

$$
c\left(X_{1} X_{2} \ldots X_{s} A\right)>c(A) .
$$

b) If $Y_{1}, Y_{2}, \cdots, Y_{t} \in \mathcal{T}_{m}$ are such that

$$
c\left(A Y_{1} Y_{2} \ldots Y_{t}\right)>c\left(A Y_{1} Y_{2} \ldots Y_{t-1}\right)>\ldots>c\left(A Y_{1} Y_{2}\right)>c\left(A Y_{1}\right)>c(A),
$$

then

$$
r\left(A Y_{1} Y_{2} \ldots Y_{t}\right)>r(A) .
$$

\section{Semi-canonical and canonical $\mathcal{M}_{n \times m}^{p}$-matrices}

Definition 3.1. Let $A \in \mathcal{M}_{n \times m}^{p}, r(A)=\left\langle x_{1}, x_{2}, \ldots, x_{n}\right\rangle$ and $c(A)=\left\langle y_{1}, y_{2}, \ldots, y_{m}\right\rangle$. We will call the matrix $A$ semi-canonical, if

$$
x_{1} \leq x_{2} \leq \cdots \leq x_{n}
$$

and

$$
y_{1} \leq y_{2} \leq \cdots \leq y_{m}
$$

Lemma 3.1. Let $A=\left(a_{s t}\right)_{n \times m} \in \mathcal{M}_{n \times m}^{p}$ be a semi-canonical matrix. Then, there exist integers $s, t$, such that $1 \leq s \leq n, 1 \leq t \leq m$ and

$$
\begin{aligned}
& a_{11}=a_{12}=\cdots=a_{1 s}=0, \quad 1 \leq a_{1, s+1} \leq a_{1, s+2} \leq \cdots \leq a_{1 m} \leq p-1 \\
& a_{11}=a_{21}=\cdots=a_{t 1}=0, \quad 1 \leq a_{t+1,1} \leq a_{t+2,1} \leq \cdots \leq a_{n 1} \leq p-1
\end{aligned}
$$


Proof. Let $r(A)=\left\langle x_{1}, x_{2}, \ldots x_{n}\right\rangle$ and $c(A)=\left\langle y_{1}, y_{2}, \ldots y_{m}\right\rangle$. We assume that there exist integers $p$ and $q$, such that $1 \leq p<q \leq m, a_{1 p} \geq a_{1 q}$. In this case $y_{p}>y_{q}$, which contradicts the condition for semi-canonicity of the matrix $A$. We have proven (6). Similarly, we prove (7) as well.

Definition 3.2. We will call the matrix $A \in \mathcal{M}_{n \times m}^{p}$ canonical matrix, if $r(A)$ is the minimal element with respect to the lexicographic order in the set $\{r(B) \mid B \sim A\}$.

Problem 3.1. For given $m, n$ and $p$, find all canonical $\mathcal{M}_{n \times m}^{p}$-matrices satisfying certain conditions.

Particular cases of Problem 3.1 are as follows:

Problem 3.2. For given $n$ and $k$, find all $n \times n$ canonical weighing matrix with weight $k$.

Problem 3.3. For given $n$, find all $n \times n$ canonical Hadamard matrices.

If the matrix $A \in \mathcal{M}_{n \times m}^{p}$ is canonical and $r(A)=\left\langle x_{1}, x_{2}, \ldots, x_{n}\right\rangle$, then obviously

$$
x_{1} \leq x_{2} \leq \ldots \leq x_{n}
$$

From Definition 3.2 it immediately follows that there exists only one canonical binary matrix in every class on the equivalence relation " " (see Definition 1.1).

Lemma 3.2. If the matrix $A \in \mathcal{M}_{n \times m}^{p}$ is a canonical matrix, then $A$ is a semi-canonical matrix.

Proof. Let $A \in \mathcal{M}_{n \times m}^{p}$ be a canonical matrix and $r(A)=\left\langle x_{1}, x_{2}, \ldots, x_{n}\right\rangle$. Then, from (8) it follows that $x_{1} \leq x_{2} \leq \cdots \leq x_{n}$. Let $c(A)=\left\langle y_{1}, y_{2}, \ldots, y_{m}\right\rangle$. We assume that there are $s$ and $t$ such that $s \leq t$ and $y_{s}>y_{t}$. Then, we swap the columns of numbers $s$ and $t$. Thus, we obtain the matrix $A^{\prime} \in \mathcal{M}_{n \times m}^{p}, A^{\prime} \neq A$. Obviously $c\left(A^{\prime}\right)<c(A)$. From Theorem 2.1 and Theorem 2.2 it follows that $r\left(A^{\prime}\right)<r(A)$, which contradicts the minimality of $r(A)$.

In the next example, we will see that the opposite statement of Lemma 3.2 is not always true.

Example 3.1. We consider the matrices:

$$
A=\left(\begin{array}{llll}
0 & 0 & 1 & 2 \\
0 & 0 & 2 & 2 \\
0 & 2 & 0 & 0 \\
1 & 0 & 0 & 0
\end{array}\right) \in \mathcal{M}_{4 \times 4}^{3}
$$

and

$$
B=\left(\begin{array}{cccc}
0 & 0 & 0 & 2 \\
0 & 1 & 2 & 0 \\
0 & 2 & 2 & 0 \\
1 & 0 & 0 & 0
\end{array}\right) \in \mathcal{M}_{4 \times 4}^{3}
$$


After immediate verification, we find that $A \sim B$. Furthermore, $r(A)=\langle 5,8,18,27\rangle$, $c(A)=\langle 1,6,45,72\rangle, r(B)=\langle 2,15,24,27\rangle, c(B)=\langle 1,15,24,54\rangle$. So $A$ and $B$ are two equivalent semi-canonical matrices, but they are not canonical. The canonical matrix in this equivalence class is the matrix

$$
C=\left(\begin{array}{llll}
0 & 0 & 0 & 1 \\
0 & 0 & 2 & 0 \\
1 & 2 & 0 & 0 \\
2 & 2 & 0 & 0
\end{array}\right) \in \mathcal{M}_{4 \times 4}^{3},
$$

where $r(C)=\langle 1,6,45,72\rangle$ and $c(C)=\langle 5,8,18,27\rangle$.

From Example 3.1 it immediately follows that there may be more than one semi-canonical element in a given equivalence class.

\section{Necessary and sufficient conditions for a $\mathcal{M}_{n \times m}^{p}$-matrix to be canonical}

Let $A=\left(a_{i j}\right) \in \mathcal{M}_{n \times m}^{p}, r(A)=\left\langle x_{1}, x_{2}, \ldots, x_{n}\right\rangle$. We introduce the following notations:

- $\nu_{i}(A)=\nu\left(x_{i}\right)=$ the number of nonzero entries in the $i$-th row of $A, i=1,2, \ldots n$.

- $Z_{i}(A)=Z\left(x_{i}\right)=\left\{x_{k} \in r(A) \mid x_{k}=x_{i}\right\}$ - the set of all rows $x_{k} \in r(A)$, such that $x_{k}=x_{i}$. By definition $x_{i} \in Z\left(x_{i}\right), i=1,2, \ldots n$.

- $\zeta_{i}(A)=\zeta\left(x_{i}\right)=\left|Z_{i}(A)\right|, i=1,2, \ldots n$.

Lemma 4.1. Let $A=\left(a_{i j}\right) \in \mathcal{M}_{n \times m}^{p}, r(A)=\left\langle x_{1}, x_{2}, \ldots, x_{n}\right\rangle$ and let $x_{1} \leq x_{2} \leq \cdots \leq x_{n}$. Then, for each $i=2,3, \ldots, n$, for which $x_{i-1}<x_{i}$, or $i=1$ the condition

$$
Z\left(x_{i}\right)=\left\{x_{i}, x_{i+1}, \ldots, x_{i+\zeta\left(x_{i}\right)-1}\right\}
$$

is fulfilled.

Proof. Trivial.

The formulation of the following theorem will help us to construct a recursive algorithm for obtaining all canonical $\mathcal{M}_{n \times m}^{p}$-matrices.

Theorem 4.2. Let $A=\left(a_{i j}\right) \in \mathcal{M}_{n \times m}^{p}, r(A)=\left\langle x_{1}, x_{2}, \ldots, x_{n}\right\rangle, c(A)=\left\langle y_{1}, y_{2}, \ldots, y_{m}\right\rangle$, $s=\nu_{1}(A), t=\zeta_{1}(A)$. Then, $A$ is canonical if and only if the following conditions are satisfied:

1. $x_{1} \leq x_{2} \leq \cdots \leq x_{n} \leq p^{m}-1$

2. $\frac{p^{s}-1}{p-1} \leq x_{1} \leq p^{s}-1$;

3. If $s>1$, then $y_{m-s+1} \leq y_{m-s+2} \leq y_{m}$; 
4. For each $i=2,3, \ldots, n, \nu_{1}(A) \leq \nu_{i}(A)$;

5. Let $t<n$. Let an integer $i$ exist such that $t<i \leq n$ and $\nu_{i}(A)=\nu_{1}(A)=s$. Then, we successively get the matrices $A^{\prime}, A^{\prime \prime}$ and $A^{\prime \prime \prime}$ in the following way:

(a) We get the matrix $A^{\prime}$ by moving the rows from the set $Z_{i}(A)$ so they become first;

(b) If $s=m$, then $A^{\prime \prime}=A^{\prime}$. Let $s<m, A^{\prime}=\left(a_{i j}^{\prime}\right)$ and let $\Upsilon=\left\{j \mid a_{1 j}^{\prime} \neq 0\right\}=$ $\left\{u_{1}, u_{2}, \ldots u_{s}\right\}$. Then, we get the matrix $A^{\prime \prime}$ by moving successively the $u_{k}$-th column $(k=1,2, \ldots, s)$ from $A^{\prime}$ so it becomes last in $A^{\prime \prime}$;

(c) We get the matrix $A^{\prime \prime \prime}$ by sorting the last s columns of $A^{\prime \prime}$ in ascending order.

Then, $r(A) \leq r\left(A^{\prime \prime \prime}\right)$.

6. Let $1 \leq t<n$ and $0 \leq s<m$. Let the matrix $B \in \mathcal{M}_{(n-t) \times(m-s)}^{p}$ be obtained from $A$ by removing the first $t$ rows and the last s columns. Then, $B$ is canonical.

Proof. Necessity. Let $A=\left(a_{i j}\right) \in \mathcal{M}_{n \times m}^{p}$ be a canonical matrix and let $r(A)=\left\langle x_{1}, x_{2}, \ldots, x_{n}\right\rangle$, $c(A)=\left\langle y_{1}, y_{2}, \ldots, y_{m}\right\rangle$.

Condition 1 follows from the fact that every canonical matrix is semi-canonical (Lemma 3.2), so $x_{1} \leq x_{2} \leq \cdots \leq x_{n}$ and from inequality (2).

From equation (1) and Lemma 3.1 it follows that

$$
x_{1}=\sum_{j=1}^{m} a_{1 j} p^{m-j}=\sum_{j=m-s+1}^{m} a_{1 j} p^{m-j} \geq \sum_{j=m-s+1}^{m} 1 \cdot p^{m-j}=\frac{p^{s}-1}{p-1}
$$

and

$$
x_{1}=\sum_{j=m-s+1}^{m} a_{1 j} p^{m-j} \leq \sum_{j=m-s+1}^{m}(p-1) p^{m-j}=(p-1) \frac{p^{s}-1}{p-1}=p^{s}-1 .
$$

Therefore, Condition 2 is true.

Condition 3 follows from the fact that every canonical matrix is semi-canonical (Lemma 3.2).

We assume that an integer $i, 2 \leq i \leq n$ exists, such that $\nu_{i}(A)<\nu_{1}(A)=s$ and let $\nu_{i}(A)=u<s$. Then, a matrix $A^{\prime}=\left(a_{i j}^{\prime}\right) \sim A$ exists such that $a_{i 1}^{\prime}=a_{i 2}^{\prime}=\cdots=a_{i m-u}^{\prime}=0$ and $1 \leq a_{i m-u+1}^{\prime} \leq a_{i m-u+2}^{\prime} \leq \cdots \leq a_{i m}^{\prime} \leq p-1$. We move the $i$-th row of $A^{\prime}$ at first place and we obtain a matrix $A^{\prime \prime}$. Obviously $A^{\prime \prime} \sim A$. Let $r\left(A^{\prime \prime}\right)=\left\langle x_{1}^{\prime \prime}, x_{2}^{\prime \prime}, \ldots, x_{n}^{\prime \prime}\right\rangle$. From the above proven Condition 2, it follows that $x_{1} \geq \frac{p^{s}-1}{p-1}=p^{s-1}+p^{s-2}+\cdot+p^{u}+p^{u-1}+\cdots+p+1>$ $p^{u}>p^{u}-1 \geq x_{1}^{\prime \prime}$. Therefore, $x_{1}>x_{1}^{\prime \prime}$, i.e., $r(A)>r\left(A^{\prime \prime}\right)$, which is impossible, due to the fact that $A$ is canonical. Thus, Condition 4 is true.

Condition 5 comes directly from the fact that $A$ is canonical and $r(A) \leq r(U)$ for each matrix $U \sim A$.

Let $t=\zeta_{1}(A)<n$ and let $s=\nu_{1}(A)<m$. From the already proved Conditions 1, 2, 4 and 5 and Lemma 4.1, it follows that $A$ is presented in the form:

$$
A=\left(\begin{array}{ll}
O & N \\
B & C
\end{array}\right)
$$


where $O$ is a $t \times(m-s)$ matrix, all elements of which are equal to $0, N$ is a $t \times s$ matrix, all elements of which are equal to each other and which are not equal to 0 and all rows of the matrix $(O N)_{t \times m}$ coincide with the elements of the set $Z_{1}(A), B \in \mathcal{M}_{(n-t) \times(m-s)}^{p}, C \in \mathcal{M}_{(n-t) \times s}^{p}$.

Let $B^{\prime} \sim B$ and let $B^{\prime}$ be a canonical $\mathcal{M}_{(n-t) \times(m-s)}^{p}$-matrix. Then, the following matrices $A^{\prime} \in \mathcal{M}_{n \times m}^{p}$ and $C^{\prime} \in \mathcal{M}_{(n-t) \times s}^{p}$ exist, such that $A^{\prime} \sim A, C^{\prime} \sim C, A^{\prime}=\left(\begin{array}{cc}O & N \\ B^{\prime} & C^{\prime}\end{array}\right)$, and $C^{\prime}$ is obtained from $C$ after an eventual permutation of the rows. Let $r\left(A^{\prime}\right)=\left\langle x_{1}^{\prime}, x_{2}^{\prime}, \ldots, x_{n}^{\prime}\right\rangle$. Obviously $x_{i}^{\prime}=x_{i}$ for all $i=1,2, \ldots$. Let us assume that $B^{\prime} \neq B$, i.e., $r\left(B^{\prime}\right)<r(B)$. Let $r(B)=\left\langle b_{t+1}, b_{t+2}, \ldots, b_{n}\right\rangle, r\left(B^{\prime}\right)=\left\langle b_{t+1}^{\prime}, b_{t+2}^{\prime}, \ldots, b_{n}^{\prime}\right\rangle, r(C)=\left\langle c_{t+1}, c_{t+2}, \ldots, c_{n}\right\rangle, r\left(C^{\prime}\right)=$ $\left\langle c_{t+1}^{\prime}, c_{t+2}^{\prime}, \ldots, c_{n}^{\prime}\right\rangle$. From assumption it follows that there exist $i \in[t+1, n]$ such that $b_{t+1}^{\prime}=b_{t+1}$, $b_{t+2}^{\prime}=b_{t+2}, \ldots, b_{i-1}^{\prime}=b_{i-1}$ and $b_{i}^{\prime}<b_{i}$, i.e., $b_{i}^{\prime}+1 \leq b_{i}$. Then, $x_{1}^{\prime}=x_{1}, x_{2}^{\prime}=x_{2}, \ldots, x_{i-1}^{\prime}=$ $x_{i-1}$. Since $0 \leq c_{k}<p^{s}$ and $0 \leq c_{i}^{\prime}<p^{s}$, for each $i \in[t+1, n]$, then $x_{i}^{\prime}=b_{i}^{\prime} p^{s}+c_{i}^{\prime} \leq$ $\left(b_{i}^{\prime}+1\right) p^{s}+c_{i}^{\prime}=b_{i} p^{s}+p^{s}+c_{i}^{\prime}+c_{i}-c_{i} \leq b_{i} p^{s}+p^{s}+p^{s}+c_{i}-0<b_{i} p^{s}+c_{i}$. Consequently $r\left(A^{\prime}\right)<r(A)$. But $A$ is canonical, i.e., $r(A) \leq r\left(A^{\prime}\right)$, which is a contradiction. Therefore, $B^{\prime}=B$ and $B$ is canonical. Thus, we have proved Condition 6 .

Sufficiency. Let $A \in \mathcal{M}_{n \times m}^{p}$ satisfy Conditions $1 \div 6$ and hence the conditions of Lemma 4.1 are fulfilled. Let $r(A)=\left\langle x_{1}, x_{2}, \ldots, x_{n}\right\rangle$ and $c(A)=\left\langle y_{1}, y_{2}, \ldots, y_{m}\right\rangle$.

If $t=n$, then $x_{1}=x_{2}=\cdots=x_{n}$ and according to Condition 3 it is easy to see that $A$ is a canonical $\mathcal{M}_{n \times m}^{p}$-matrix.

If $t<n$ and $s=m$, then according to Condition 1, Lemma 4.1 and Conditions 4 and 5 it is easy to see that $A$ is a canonical $\mathcal{M}_{n \times m}^{p}$-matrix.

Let $1 \leq t<n$ and $0 \leq s<m$. Let $U \sim A$ and let $U$ be a canonical $\mathcal{M}_{n \times m}^{p}$-matrix. Since the Conditions $1 \div 6$ are necessary for the canonicity of a matrix, consequently $U$ also satisfies these conditions. According to Condition 4,

$$
\nu_{1}(U)=\nu_{1}(A)=s
$$

Thus, the matrix $U$ is represented in the form (9) and let

$$
A=\left(\begin{array}{ll}
O & N \\
B & C
\end{array}\right) \text { and } U=\left(\begin{array}{cc}
O^{\prime} & N^{\prime} \\
B^{\prime} & C^{\prime}
\end{array}\right),
$$

Let us assume that $U$ is obtained from $A$ only by permutation of the columns. In this case obviously $\zeta_{1}(U)=\zeta_{1}(A)=t, \nu_{1}(U)=\nu_{1}(A)=s, O^{\prime}=O, N^{\prime} \sim N, B^{\prime} \sim B$ and $C^{\prime} \sim C$.

Permutation of columns which are different each other and which belong only to the set $Y_{1}=\left\{y_{1}, y_{2}, \ldots, y_{m-s}\right\}$ without permutation of different each other rows is impossible in accordance with Condition 6.

Permutation of columns, which are different from each other and which belong only to the set $Y_{2}=\left\{y_{m-s+1}, y_{m-s+2}, \ldots, y_{m}\right\}$ without permutation of mutually different rows, is impossible in accordance with Condition 3.

Therefore, there are $k, l$ such that $1 \leq k \leq m-s<l \leq m$ and the $k$-th column has become the $l$-th, or the $l$-th column has become the $k$-th. Then, according to Condition 3 and equation (9) easily see that it is impossible if we did not change the places of some rows. 
Therefore, $U$ is obtained from $A$ by swapping some of the rows. Without loss of generality, we can assume that $U$ is obtained from $A$ in the beginning by swapping some rows, then (if it is necessary) swapping some columns.

Permutation of rows that belong only to the set $X_{1}=\left\{x_{1}, x_{2}, \ldots, x_{t}\right\}=Z_{1}(A)$ does not change the matrix $A$ because $x_{1}=x_{2}=\ldots=x_{t}$.

Permutation of rows that belong only to the set $X_{2}=\left\{x_{t+1}, x_{t+2}, \ldots, x_{n}\right\}$ is impossible in accordance with Condition 6.

Therefore, taking into account the Conditions 1 and 4 and Lemma 4.1, we conclude that we have changed the first $t=\zeta_{1}(A)$ rows with another equal to each rows of the set $Z_{j}(A)$, $t+1 \leq j \leq n$. After that, in order to obtain a matrix of kind (9), if it is necessary, we have to change the places of some columns of the matrix $A$. According to Conditions 3 and 5 it follows that $r(A) \leq r(U)$. But $U$ is canonical, i.e., $r(U) \leq l(A)$. Therefore, $U=A$, i.e., $A$ is canonical.

\section{References}

[1] Best, D., \& Kharaghani, H. (2010) Unbiased complex hadamard matrices and bases. Cryptography and Communications, 2 (2), 199-209.

[2] Hedayat, A., \& Wallis, W. D. (1978) Hadamard matrices and their applications. The Annals of Statistic, 6 (6), 1184-1238.

[3] Horadam, K. J. (2007) Hadamard Matrices and Their Applications. Princeton University Press, Princeton, New Jersey.

[4] Koukouvinos, C., \& Seberry, J. (1997) Weighing matrices and their applications. Journal of Statistical Planning and Inference, 62 (1), 91-101.

[5] Sachkov, V. N., \& Tarakanov, V. E. (2002) Combinatorics of Nonnegative Matrices. Translations of Mathematical Monographs. American Mathematical Society.

[6] Tarakanov, V. E. (1985) Combinatorial Problems and (0,1)-matrices. Nauka, Moscow (in Russian).

[7] Yordzhev, K. (2017) On the cardinality of a factor set of binary matrices. Linear Algebra and Its Applications, 534, 122-134. 